\title{
Low-energy electron collisions with gas-phase uracil
}

\author{
Carl Winstead and Vincent McKoy \\ A. A. Noyes Laboratory of Chemical Physics, California Institute of Technology, Pasadena, \\ California 91125
}

(Received 26 June 2006; accepted 16 August 2006; published online 6 November 2006)

\begin{abstract}
We have studied gas-phase collisions between slow electrons and uracil molecules with a view to understanding the resonance structure of the scattering cross section. Our symmetry-resolved results for elastic scattering, computed in the fixed-nuclei, static-exchange and static-exchange-plus-polarization approximations, provide locations for the expected $\pi^{*}$ shape resonances and indicate the possible presence of a low-energy $\sigma^{*}$ resonance as well. Electron-impact excitation calculations were carried out for low-lying triplet and singlet excitation channels and yield a very large singlet cross section. We discuss the connection between the resonances found in our elastic cross section and features observed in dissociative attachment. (C) 2006 American Institute of Physics. [DOI: 10.1063/1.2353147]
\end{abstract}

\section{INTRODUCTION}

The interaction of DNA, RNA, and their constituents with slow electrons has been a topic of considerable recent interest. Seminal experiments by Sanche and co-workers ${ }^{1,2}$ demonstrated that slow electrons can produce single- and double-strand breaks in DNA and are therefore genotoxic. This discovery inspired numerous experiments aimed at elucidating various aspects of the electron-DNA interaction. Gas-phase experiments ${ }^{3-22}$ have explored electron collisions with molecular subunits including the purine and pyrimidine bases of DNA, ${ }^{3,5-7,10-13,15-22}$ the RNA base uracil, $7,10,11,13,17-19,21,22$ and halogenated derivatives. $5,8-10,14,17$ Most of these experiments have studied dissociative attachment ${ }^{3,5-7,10-13,15-22}$ and/or electron-impact excitation and ionization. ${ }^{8,9,12-15}$ However, Burrow and co-workers have carried out electron transmission measurements ${ }^{4,10}$ to determine the energies of scattering resonances, and they have assigned the observed features as $\pi^{*}$ shape resonances based on computed orbital energies and energy shifts determined from related molecules.

A major question is the relationship, if any, between narrow, apparently resonant features that are seen in low-energy dissociative attachment and shape resonances in the elastic cross section. In uracil, for example, a strong feature is seen at $1.0 \mathrm{eV}$ in the production cross section for $(\mathrm{U}-\mathrm{H})^{-}$(that is, the anion formed after removing one hydrogen from uracil), $7,10,11,13,17-19,22$ with weaker features at 0.7 and possibly $0.8 \mathrm{eV}$. It is natural to suspect the involvement of a shape resonance; however, these energies do not coincide with any of the $\pi^{*}$ resonance assignments of Burrow and co-workers, ${ }^{4,10}$ and an alternative explanation in terms of vibrational Feshbach resonances built on the dipole-bound anion has been proposed. ${ }^{10,19,22}$ On the other hand, a broader peak in the dissociative attachment spectrum near $2 \mathrm{eV}$ has been attributed to a $\pi^{*}$ resonance. ${ }^{22}$

Simons and co-workers ${ }^{23-26}$ have used bound-state methods on subunits of DNA to investigate possible resonance-mediated mechanisms for causing strand breaks, but few calculations have been performed using scattering methods. Możejko and Sanche ${ }^{27}$ computed elastic electron cross sections for uracil and the four DNA bases, but their results were restricted to energies of $50 \mathrm{eV}$ and above because of the approximations made. Gianturco and Lucchese ${ }^{28}$ reported $\pi^{*}$ and $\sigma^{*}$ shape-resonance energies determined from a scattering calculation; however, Burrow has questioned $^{29}$ the correctness of these results on both experimental and theoretical grounds. Grandi and co-workers ${ }^{30}$ also reported a study of uracil at higher collision energies, $\sim 9 \mathrm{eV}$, using a similar approach to that of Gianturco and Lucchese but with the further approximation of imposing $D_{2 h}$ symmetry on the scattering electron's wave function. Gianturco and Lucchese do not report cross sections, and Grandi and co-workers report only partial cross sections for some $\left(D_{2 h}\right)$ symmetries. Very recently, Tonzani and Greene ${ }^{31}$ reported low-energy elastic electron scattering cross sections, including $\pi^{*}$ resonance energies, for the four DNA bases and the RNA base uracil. The resonance positions differ from those of Gianturco and Lucchese ${ }^{28}$ and also from the electron-transmission measurements. ${ }^{4,10}$ All of the existing calculations employ local approximations to the exchange and polarization interactions, which strongly influence lowenergy electron scattering, in order to reduce the problem to one of potential scattering.

In the present work, we apply the Schwinger multichannel (SMC) method, ${ }^{32}$ an all-electron formulation that does not rely on local-potential approximations, to the elastic and inelastic scattering of low-energy electrons by uracil. As will be seen, our elastic calculations yield $\pi^{*}$ resonance positions that do not entirely agree with the reported experimental positions, but they nonetheless appear to be generally supportive of the assignments of Burrow and co-workers; ${ }^{4,10}$ in particular, they agree with the experimental assignments on one important point, the location of the lowest $\pi^{*}$ resonance in the $\sim 0.2-0.3 \mathrm{eV}$ energy range. The elastic calculations also indicate the possible presence of a low-energy $\sigma^{*}$ shape resonance. In addition to the elastic results, we report two- and three-channel calculations for electron-impact excitation of 
TABLE I. Nuclear coordinates used in all scattering calculations (bohr units).

\begin{tabular}{lccc}
\hline \hline Atom & $x$ & $y$ & $z$ \\
\hline $\mathrm{N}$ & 0.059006 & -1.792536 & 0.0 \\
$\mathrm{C}$ & -2.317973 & -0.697014 & 0.0 \\
$\mathrm{C}$ & 2.441760 & -0.607576 & 0.0 \\
$\mathrm{O}$ & -4.303301 & -1.883598 & 0.0 \\
$\mathrm{O}$ & 4.386475 & -1.871878 & 0.0 \\
$\mathrm{~N}$ & -2.212165 & 1.927220 & 0.0 \\
$\mathrm{C}$ & 2.286967 & 2.141239 & 0.0 \\
$\mathrm{C}$ & 0.004483 & 3.288567 & 0.0 \\
$\mathrm{H}$ & 0.078648 & -3.714247 & 0.0 \\
$\mathrm{H}$ & -3.914890 & 2.802138 & 0.0 \\
$\mathrm{H}$ & -0.215196 & 5.327645 & 0.0 \\
$\mathrm{H}$ & 4.027749 & 3.213659 & 0.0 \\
\hline \hline
\end{tabular}

two important electronic states of uracil: the lowest $(\pi$ $\left.\rightarrow \pi^{*}\right)$ triplet state, whose vertical threshold is much lower than that of any other excited state, and the lowest $(\pi$ $\left.\rightarrow \pi^{*}\right)$ singlet state, which carries a large oscillator strength from the ground state and therefore can be expected to have a large electron-impact cross section as well.

\section{COMPUTATIONAL DETAILS}

The SMC method and its implementation have been described elsewhere, ${ }^{32-34}$ so here we present only those details specific to the present study of uracil.

The uracil molecule exists in several tautomers. We carried out our scattering calculations for the diketo tautomer, which is the lowest-energy conformation in the gas phase. The nuclear geometry was optimized within the $\mathrm{C}_{s}$ point group using the electronic structure program GAMESS (Ref. 35) at the level of second-order Möller-Plesset perturbation theory within the $6-31 \mathrm{G}(d)$ basis set. The resulting nuclear coordinates are listed in Table I.

For the elastic scattering calculations, we made an extensive exploration of the use of different basis sets and different treatments of polarization. Uracil presents unusual numerical difficulties not only because of its size and low symmetry but also because of its large dipole moment, $\sim 4$ -5 debye ${ }^{36-38}$ which is sufficient to form at least one dipolebound anion state s6,39-44 $^{3}$ and which will strongly influence the scattering cross section at low energies. Indeed, the fixed-nuclei elastic scattering cross section for a polar molecule is formally divergent. ${ }^{45}$ Although this difficulty can be surmounted, ${ }^{45}$ we have simply ignored it in the present work. It is possible to do so because our use of finite, squareintegrable basis sets effectively truncates the partial-wave expansion of the scattering wave function and thus precludes divergence of the cross section. Moreover, at the very lowest energies where correcting the fixed-nuclei cross section for high-partial-wave scattering is most critical, neglect of vibration is a poor approximation, and the cross section could not be considered quantitative even after correction. At any rate, we do not expect the details of high-partial-wave, largeimpact-parameter scattering to have much effect on the resonance positions that are our principal interest.
Although we do not make an explicit correction for dipolar scattering, we do hope to obtain semiquantitative results except at the very lowest energies, and doing so in the presence of a large dipole moment requires use of an extended one-electron basis set, including diffuse functions and functions centered away from the nuclei. We are able to use quite large basis sets for static-exchange (SE) calculations, but current program limitations restrict the basis size for static-exchange-plus-polarization (SEP) calculations, which appears to affect numerical stability in the presence of a large dipole. Moreover, the use of large, diffuse basis sets itself can lead to problems with numerical linear dependence, including spurious resonances. Extra care must thus be taken in the interpretation of results. Comparison among calculations using different basis sets and different treatments of polarization helps to distinguish between spurious and physical resonances.

The low symmetry and large size of uracil also pose challenges in the treatment of polarization effects. In the SMC method, polarization is represented by virtual excitations of the target molecule's (Hartree-Fock) ground-state wave function. With such a representation, the number of $(N+1)$-electron configurations needed to describe the $N$-electron target plus the projectile electron scales formally as $N^{3}$, meaning that storage for dense square matrices defined in the configuration space will scale as $N^{6}$ while the work to invert or diagonalize such matrices will scale as $N^{9}$. This rapid scaling puts a high premium on practical means of controlling the size of the configuration space. In the fixednuclei approximation, the overall electronic wave function symmetry is conserved during the collision, so the evaluation of the cross section can be partitioned into independent calculations for each irreducible representation, leading to tremendous savings in high-symmetry molecules. Physical insight can also be used to restrict the number of virtual excitations. Excitations out of core orbitals can generally be omitted with little loss of accuracy, while transformations can be applied to the virtual-orbital space to produce compact subsets of orbitals that are particularly effective as "particle" orbitals. ${ }^{46-50}$ However, the $C_{s}$ point group of uracil contains only two irreducible representations; thus, it is only possible to decompose the calculation into two pieces, and it is more difficult to separate resonant from nonresonant background scattering. Moreover, the number of valence "hole" orbitals remains large even after core-hole excitations are excluded. Current program limitations restrict us to fewer than $2^{14}(16384)(N+1)$-electron configurations per irreducible representation (though we expect to relax that limit considerably in future work). We therefore cannot use as extensive a treatment of polarization (in terms of the size of the particle space) for uracil as we found to be necessary to obtain well-converged differential cross sections in recent calculations on smaller molecules. ${ }^{49,50}$ On the other hand, we are principally interested in the $\pi^{*}$ resonance positions and the integral cross section, each of which we expect to converge more quickly, especially because the long-range electron-target potential is dominated by the large static dipole rather than by the charge-induced-dipole (polarization) interaction. 
TABLE II. Supplementary Cartesian Gaussian functions used in basis B.

\begin{tabular}{cl}
\hline \hline Type & Exponent \\
\hline$s$ & 0.1 \\
$s$ & 0.03 \\
$s$ & 0.01 \\
$s$ & 0.003 \\
$p$ & 0.3 \\
$p$ & 0.1 \\
$p$ & 0.03 \\
$p$ & 0.003 \\
\hline
\end{tabular}

With the above considerations in mind, our treatment of polarization in overall ${ }^{2} A^{\prime \prime}$ symmetry (the symmetry of the $\pi^{*}$ resonances) is as follows. We include all ${ }^{2} A^{\prime \prime}(N+1)$ electron configuration state functions (CSFs) formed by antisymmetrizing ${ }^{1} A^{\prime}$ virtual excitations from valence orbitals with three compact, $\pi^{*}$-like orbitals, which are chosen as the three lowest $a^{\prime \prime}$ modified virtual orbitals (MVOs) (Ref. 51) obtained from a +4 cationic Fock operator. We further include all additional ${ }^{2} A^{\prime \prime}$ CSFs that can be formed by antisymmetrizing ${ }^{1} A^{\prime}$ or ${ }^{3} A^{\prime}$ excitations out of the highest occupied molecular orbital into one of the three " $\pi^{*}$ " MVOs with any of the $a^{\prime \prime}$ virtual orbitals. This set of configuration is intended to represent well the relaxation of the target in the presence of an electron temporarily trapped in a $\pi^{*}$ shape resonance, and therefore, it is hoped, to produce good $\pi^{*}$ resonance energies. We present below results obtained in three different one-electron basis sets. The first, basis A, is just the 6-311 $++\mathrm{G}(d, p)$ basis set as defined in GAMESS, ${ }^{35}$ which for uracil contains 212 Cartesian Gaussians and, after excluding the $x^{2}+y^{2}+z^{2}$ " $3 s$ " combination of Cartesian $d$ functions, 204 molecular orbitals. Basis B is formed by supplementing the $6-311++\mathrm{G}(d, p)$ basis with the diffuse functions listed in Table II centered both at the origin, which roughly coincides with the center of the ring, and at $+4.8 \AA$ on the $y$ axis (the positive end of the molecule), for a total of 244 Cartesian Gaussians and 236 molecular orbitals. Basis $\mathrm{C}$ includes the $\operatorname{TZV}(2 d, p)$ set internal to GAMESS together with a supplement comprising distributed $s$ Gaussians and diffuse functions at the positive end of the molecule, listed in Table III, for a total of 279 contracted Gaussians and 263 molecular orbitals.

For the polarization calculation in $A^{\prime}$ symmetry, we used basis $B$ and included all singlet-coupled excitations out of the 16 highest occupied molecular orbitals into the 30 lowest MVOs to form hole-particle pairs that were then coupled with the lowest $62 \mathrm{MVOs}$ to form ${ }^{2} A^{\prime}$ configuration state functions (CSFs) describing closed channels. The openchannel space included all $a^{\prime}$ virtual orbitals coupled to the Hartree-Fock ground state. The total number of ${ }^{2} A^{\prime}$ CSFs included in the calculation was 12617.

For comparison purposes, we also present below elastic cross sections obtained in the SE approximation (that is, neglecting polarization) using two different one-electron basis sets. As a baseline, we show results obtained in the 6-311 $++\mathrm{G}(d, p)$ basis set (basis A), while final values are computed in a much larger basis set, basis D, which should pro-
TABLE III. Supplementary Cartesian Gaussian functions used in basis C. Center coordinates are specified in bohr units.

\begin{tabular}{|c|c|c|c|c|}
\hline \multicolumn{3}{|c|}{ Center } & \multirow[b]{2}{*}{ Type } & \multirow[b]{2}{*}{ Exponent } \\
\hline$x$ & $y$ & $z$ & & \\
\hline 0.0 & -7.0 & \pm 2.5 & $s$ & 0.111 \\
\hline \pm 5.0 & -7.0 & \pm 2.5 & $s$ & 0.111 \\
\hline \pm 2.5 & -3.37794 & \pm 2.5 & $s$ & 0.111 \\
\hline \pm 7.5 & -3.37794 & \pm 2.5 & $s$ & 0.111 \\
\hline 0.0 & 1.0 & \pm 2.5 & $s$ & 0.111 \\
\hline \pm 5.0 & 1.0 & \pm 2.5 & $s$ & 0.111 \\
\hline \pm 10.0 & 1.0 & \pm 2.5 & $s$ & 0.111 \\
\hline \pm 2.5 & 5.0 & \pm 2.5 & $s$ & 0.111 \\
\hline \pm 7.5 & 5.0 & \pm 2.5 & $s$ & 0.111 \\
\hline 0.0 & 9.0 & \pm 2.5 & $s$ & 0.111 \\
\hline \pm 5.0 & 9.0 & \pm 2.5 & $s$ & 0.111 \\
\hline \pm 7.49885 & 9.0 & 0.0 & $s$ & 0.111 \\
\hline 0.0 & 11.5 & 0.0 & $s$ & 0.111 \\
\hline 0.0 & 11.5 & 0.0 & $s$ & 0.03 \\
\hline 0.0 & 11.5 & 0.0 & $s$ & 0.01 \\
\hline 0.0 & 11.5 & 0.0 & $s$ & 0.003 \\
\hline 0.0 & 11.5 & 0.0 & $p$ & 0.003 \\
\hline
\end{tabular}

duce results closer to the SE limit. Basis D includes, in addition to the $\operatorname{TZV}(3 d, 2 p)$ basis set of GAMESS (with default splitting factors for the $d$ and $p$ polarization functions), both diffuse orbitals centered at the positive end of the molecule, where trapping by the dipole potential may take place, and orbitals distributed over centers surrounding the molecule that improve the description of the scattering wave function at larger distances. The diffuse supplement consists of the functions shown in Table II centered at $+4.8 \AA$ on the $y$ axis, while the distributed set includes $486 s$ Gaussians, each with exponent 0.35 , on a cubic grid of centers with spacing $0.74 \AA$ that extends from -2.96 to $+2.96 \AA$ in $x$ and $y$ and from -2.22 to $+2.22 \AA$ in $z$, but with the centers in the molecular plane $(z=0)$ omitted. Basis D thus includes a total of 794 contracted Gaussians. From these we form 770 molecular orbitals after excluding the $3 s$ linear combination of Cartesian $d$ orbitals, 29 occupied and the remaining 741 available to describe the scattering electron.

Electron-impact excitation cross sections were computed for the lowest excited states of $A^{\prime}$ symmetry, $1^{3} A^{\prime}$ and $2^{1} A^{\prime}$. Single-excitation configuration-interaction (SECI) calculations using Gaussian (Ref. 52) and its internal 6-311 $++\mathrm{G}(d, p)$ basis set indicate that these states are well described by a single-configuration picture, in which they are $\pi \rightarrow \pi^{*}$ excitations from the highest occupied to the lowest unoccupied valence orbital. Our scattering calculations employed a single-configuration description, with the $\pi^{*}$ orbital represented by the triplet-coupled improved virtual orbital (IVO) (Ref. 53) for both the triplet and singlet channels and all other occupied orbitals taken from the ground-state Hartree-Fock wave function. Final results were computed using the same basis set as for the largest of the $A^{\prime \prime}$ polarization calculations, that is, the $\operatorname{TZV}(2 d, p)$ set with the supplement shown in Table III. Below the $2{ }^{1} A^{\prime}$ threshold, we used a two-channel approximation including only the ground and $1^{3} A^{\prime}$ states, and above the singlet threshold we used a three- 
channel approximation. The thresholds themselves, computed using the triplet IVO orbital, were $4.15 \mathrm{eV}$ for the $1^{3} A^{\prime}$ state and $7.73 \mathrm{eV}$ for the $2^{1} A^{\prime}$ state, reasonably close to the SECI values of 3.41 and $6.42 \mathrm{eV}$. More sophisticated calculations give thresholds of $4.00 \mathrm{eV}^{37} 3.68 \mathrm{eV}^{54}$ and $3.63 \mathrm{eV}$ (Ref. 55) for $1^{3} A^{\prime}$ and $6.28 \mathrm{eV}^{37} 5.00 \mathrm{eV},{ }^{38}$ and $5.44 \mathrm{eV}$ (Ref. 54) for $2^{1} A^{\prime}$, indicating a fairly large error in our singlet threshold.

\section{RESULTS AND DISCUSSION}

\section{A. Elastic $\pi^{*}$ resonances}

Calculations in restricted basis sets can provide a useful guide to the nature and location in energy of (valence) shape resonances, although the resulting orbital energies are typically above the resonance positions. As mentioned earlier, Burrow and co-workers assigned the $\pi^{*}$ resonances of uracil and the DNA bases using computed orbital energies and empirical energy shifts. Similarly, in our own work, we have made frequent use of minimal-basis-set orbital energies as a guide to the resonance structure. For uracil, we find that RHF calculations using the MINI minimal basis $\operatorname{set}^{56}$ put the first three virtual orbitals, all of which are $A^{\prime \prime}\left(\pi^{*}\right)$, at 3.62, 5.56, and $9.48 \mathrm{eV}$. These energies form probable upper bounds for the shape-resonance energies in our SE calculations, all of which employ much more extensive basis sets. The inclusion of the net-attractive polarization interaction in our SEP calculations should shift the resonance energies even lower.

With these energies in mind, we turn to Fig. 1, which shows the integral elastic cross sections obtained in the static-exchange approximation. At the lowest energies, the results in the larger basis set do a better job of capturing the rise in the cross section due to dipolar scattering, especially in $A^{\prime \prime}$ symmetry. Though not shown in the figure, the integral cross section in the larger basis rises to over 2.4 $\times 10^{-13} \mathrm{~cm}^{2}$ at $0.01 \mathrm{eV}$. At the highest energies shown, the larger basis set also produces a larger cross section, probably because it provides an improved representation of highpartial-wave scattering. However, the agreement between the two calculations on the positions and widths of the peaks in the $A^{\prime \prime}$ component of the cross section is rather good. A nonlinear fit to the $A^{\prime \prime}$ eigenphase sum in the larger basis set, using a cubic-polynomial background and assuming three resonances, yields resonance positions of 2.08, 4.2, and 8.2 $\mathrm{eV}$, with the respective widths being $0.23,0.44$, and $3.0 \mathrm{eV}$. These resonance positions differ from the MINI $\pi^{*}$ orbital energies by $1.5,1.6$, and $1.3 \mathrm{eV}$, respectively. Because these energy shifts are plausible in magnitude and fairly consistent, we can be quite confident in identifying the $A^{\prime \prime}$ cross-section peaks as $\pi^{*}$ shape resonances. The $A^{\prime}$ component of the static-exchange cross section, unlike the $A^{\prime \prime}$ component, does not exhibit any narrow peaks, only a broad maximum at about $11 \mathrm{eV}$. However, it is possible that weak and/or broad $\sigma^{*}$ resonances could be hidden in the very large nonresonant $A^{\prime}$ background.

Use of a very large one-electron Gaussian basis set naturally raises the possibility of linear dependence. Moreover, Schwinger-type methods are susceptible to spurious resonances arising from an eigenvalue of the $V-V G^{(+)} V$ operator
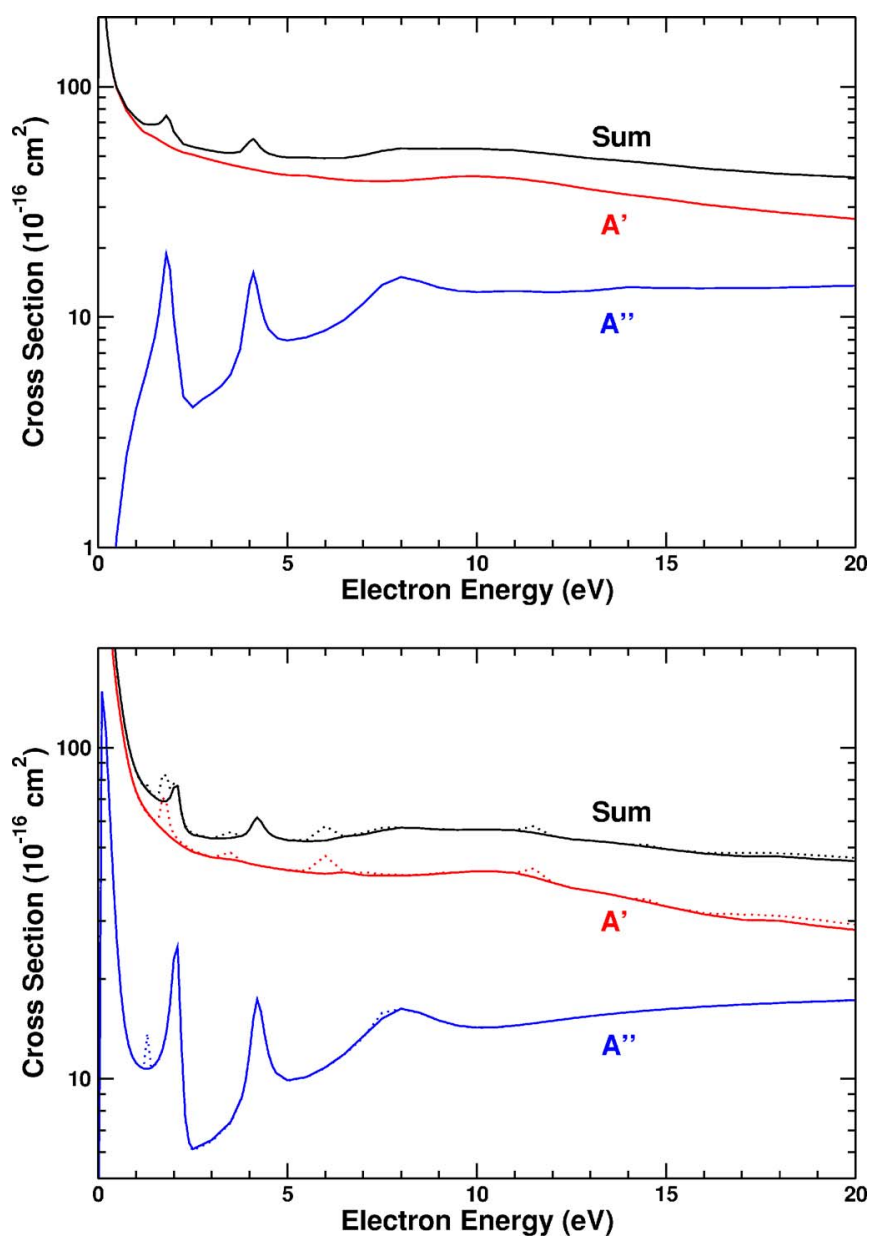

FIG. 1. (Color online) Integral elastic cross sections for electron-uracil scattering computed in the static-exchange approximation using the smaller basis set A (top) and the larger basis set D (bottom; see the text for discussion). The dotted curves in the bottom panel are results obtained with no attempt to control numerical singularity.

passing through zero. ${ }^{57}$ Accordingly, to provide some information on the question of numerical stability, the dotted curves in Fig. 1 show the results obtained by direct solution of the SMC linear equations without any attempt to control numerical singularity. The final results, shown by the solid curves, are obtained by excluding the vector associated with the smallest singular value from each of the $A^{\prime}$ and the $A^{\prime \prime}$ spaces when solving the linear systems via singular-value decomposition, thus reducing the total size of the variational space from 741 to 739 . Clearly several structures are removed, most of them in $A^{\prime}$, but comparison among the results of multiple calculations, in a variety of basis sets, indicates that the structures removed are basis-set dependent and therefore very likely spurious.

In Fig. 2, we show $A^{\prime \prime}$ results obtained with polarization included. As expected, the $\pi^{*}$ resonances shift downward in energy compared to their static-exchange positions. There is clearly some variation in the cross sections with basis set, but on the whole, the results obtained in the three different basis sets are quite consistent. We determined resonance positions and widths for basis $\mathrm{C}$ from a nonlinear least-squares fit to the $A^{\prime \prime}$ eigenphase sum in the energy range $0.13-10.0 \mathrm{eV}$, with the fitting function comprising four arctangent terms 


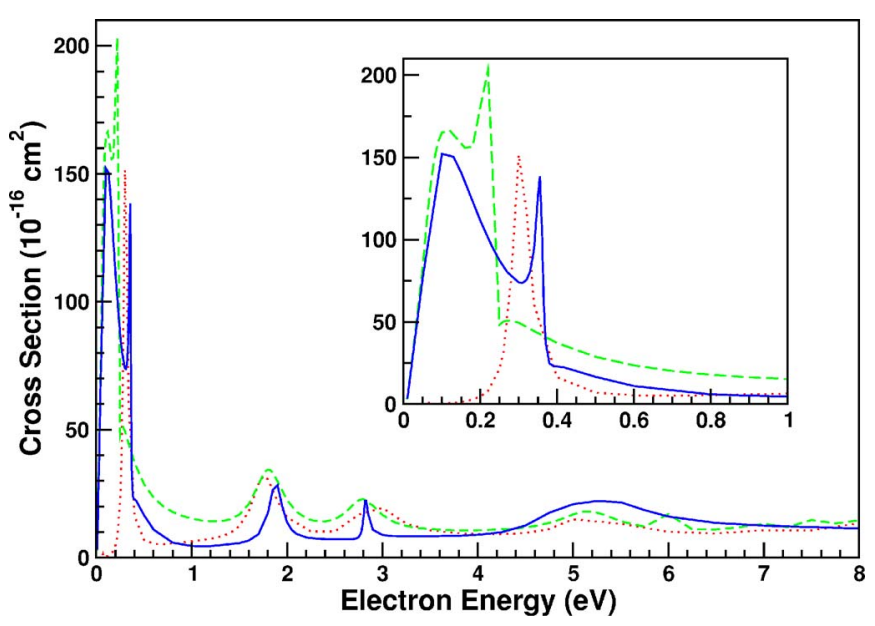

FIG. 2. (Color online) The $A^{\prime \prime}$ component of the cross section for elastic scattering of electrons by uracil computed with polarization effects included. Results are shown for three different one-electron basis sets described in the text: basis A (dots), basis B (dashes), and basis C (solid).

and a cubic polynomial to account for the background. The lowest-energy resonance is located at $0.32 \mathrm{eV}$ and appears in Fig. 2 as a narrow peak (width $0.018 \mathrm{eV}$ ) superimposed on the nonresonant maximum associated with dipolar scattering. The remaining two resonances occur at 1.91 and $5.08 \mathrm{eV}$ and are broader, with respective widths of 0.16 and $0.40 \mathrm{eV}$. The relatively large fitting error near the highest resonance makes its energy and width somewhat less certain; a second fit in the range 3.5-6.5 eV, with a quadratic background and a single resonance term, gives $5.06 \mathrm{eV}$ for the energy and 0.56 $\mathrm{eV}$ for the width. Comparing the energies determined with polarization included to the static-exchange resonance energies, we find that they are shifted downward by about 1.8, 2.3 , and $3.1 \mathrm{eV}$. These shifts are within the range $(\sim 1$ $-4 \mathrm{eV})$ that we would have anticipated based on experience with many other molecules, and thus appear reasonable.

The resonances observed in electron transmission ${ }^{4,10}$ and assigned as $\pi^{*}$ occur at $0.22,1.58$, and $3.83 \mathrm{eV}$. Agreement is therefore quite good for the position of the first resonance ( $0.10 \mathrm{eV}$ error), but our calculation places the remaining two resonances 0.33 and $1.3 \mathrm{eV}$, respectively, too high. Although we expect that we could lower the energies of these higher resonances by including more closed-channel terms in our treatment of polarization, we know of no clear criteria for choosing a closed-channel space that is larger but not too large (which might place the resonances too low). In light of the limitations of the calculation, the increasing disagreement with experiment at higher energies is perhaps not surprising. Moreover, even with those limitations, our calculations, in contrast to earlier work, ${ }^{28,31}$ appear to support the assignments made by Burrow and co-workers. In particular, we place the lowest-energy $A^{\prime \prime}$ resonance at $0.32 \mathrm{eV}$ rather than at $2.2 \mathrm{eV}^{28,31}$ This position is consistent not only with experiment but also with the upper bounds set by the minimal-basis-set and static-exchange results that were discussed above.

A puzzling feature of the cross section shown in Fig. 2 is the "extra" resonance at $2.8 \mathrm{eV}$, which does not appear to correlate with any of the minimal-basis-set orbitals, static-

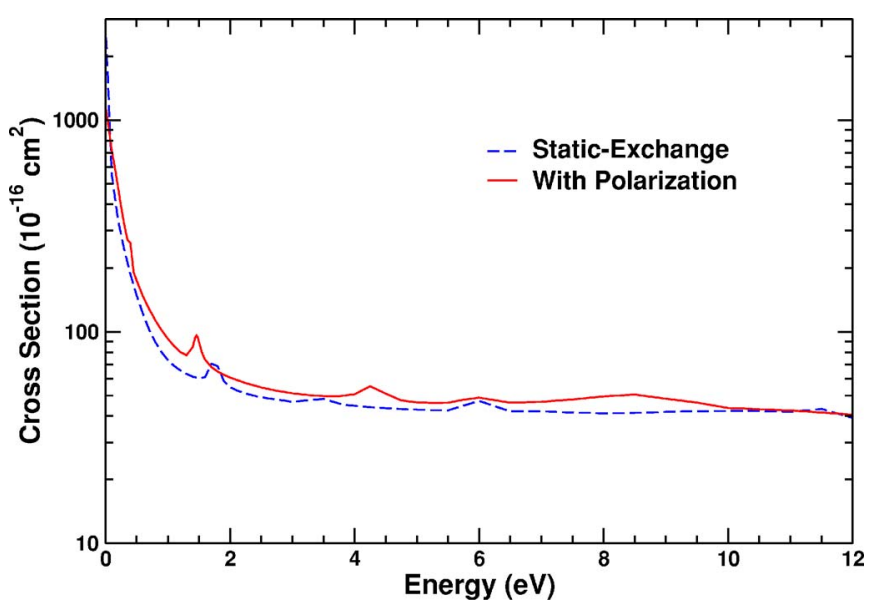

FIG. 3. (Color online) $A^{\prime}$ component of the integral cross section for elastic scattering of electrons by uracil. The solid line is the result including polarization; the dashed line is the static-exchange result computed in the same basis set.

exchange resonances, or electron-transmission resonances. It is tempting to dismiss this feature as spurious, since Schwinger-type methods are susceptible to spurious resonances that are purely numerical in origin. ${ }^{57}$ However, as the figure demonstrates, a feature persists at this energy (though with varying width) in different polarization calculations using different basis sets, and its persistence suggests that it may have a physical origin. The absence of any corresponding feature in the static-exchange cross section, even when employing an extended basis set (Fig. 1, bottom), appears to rule out any type of shape resonance, whether built upon an unoccupied molecular orbital or otherwise. Yet the extra peak also appears to fall too far below the lowest electronic threshold to be plausibly assigned as a Feshbach resonance, though we might speculate that the large dipole potential in uracil could conceivably lead to unusually strong binding of a Feshbach resonance. Because our current methods of solving large polarization problems extract scattering amplitudes without obtaining the corresponding scattering wave functions, we cannot further analyze this feature or even rule out the possibility that it is one of the $\pi^{*}$ resonances, although comparison with the static-exchange resonance positions appears to disfavor that possibility.

\section{B. $\boldsymbol{A}^{\prime}$ elastic scattering}

The $A^{\prime}$ component of the integral elastic cross section is shown in Fig. 3. For comparison, we show both the final result obtained with polarization included and the staticexchange result obtained in the same basis set. Apart from an overall enhancement of the cross section below $\sim 10 \mathrm{eV}$, there is little qualitative change in the cross section when polarization is included. Peaks in the static-exchange cross section at about 3.5 and $6 \mathrm{eV}$ are likely spurious, as are those at about 4.5 and $6 \mathrm{eV}$ in the cross section with polarization, since they are narrow and do not show the expected correlation (that is, a one-to-one correspondence between the polarized and static-exchange peaks, with the polarized results shifted downward in energy). However, the broad peak that is centered around $10.5 \mathrm{eV}$ without polarization and around 
$8.5 \mathrm{eV}$ with polarization is likely real and due to one or more $\mathrm{C}-\mathrm{H}$ or $\mathrm{N}-\mathrm{H} \sigma^{*}$ shape resonances. Mass-resolved dissociative-attachment measurements at higher energies ${ }^{7,6,13}$ reveal one or more peaks in the $\sim 5-12 \mathrm{eV}$ energy range, depending on the anion being detected, while measurements of the total ion yield ${ }^{18,19}$ show a bimodal peak at $\sim 6 \mathrm{eV}$, with possible higher-energy anion peaks obscured by the copious cation signal above the ionization potential. The strongest features, however, appear to occur at $\sim 6-7$ (Refs. 6, 13, 18, and 19) and $9 \mathrm{eV}^{6,13}$ The latter might be associated with the broad $A^{\prime}$ feature we observe centered near $8.5 \mathrm{eV}$ in our elastic cross section. Though the former coincides fairly closely with the peak we see at about $6 \mathrm{eV}$, that feature of our cross section is, as already noted, likely spurious. In both cases, it is important to note that core-excited resonances may be invoked as an alternative to elastic shape resonances to explain the features in the dissociative-attachment cross sections.

Two intriguing features are seen at low energy. At very low energy, there is a "kink" in the cross section obtained with polarization whose origin is unclear. It is associated with a small increase in the eigenphase sum and a simultaneous change in its energy dependence from sharply falling to nearly constant. At about $1.45 \mathrm{eV}$, there is a narrow peak in the cross section obtained with polarization that is associated with a jump of $\sim \pi$ in the $A^{\prime}$ eigenphase sum and that appears to correlate, as well, with the static-exchange peak at about $1.75 \mathrm{eV}$. We may therefore tentatively assign this 1.45 $\mathrm{eV}$ peak as a $\sigma^{*}$ shape resonance. The RHF calculation in the MINI minimal basis set places the lowest $a^{\prime}$ valence virtual at $13.2 \mathrm{eV}$, which appears to be too far away in energy to account for this peak, so it is possible that it arises instead from a nonvalence, diffuse state trapped by the dipole potential. On the other hand, Scheer and co-workers predict a valence $\sigma^{*}$ resonance at $\sim 2.4 \mathrm{eV}$, fairly close to our peak position, on the basis of scaled $6-31 \mathrm{G}^{*}$ virtual-orbital energies. ${ }^{10}$ Indeed, the mechanism they propose for the dissociative-attachment maximum at $1.0 \mathrm{eV}$ requires an avoided crossing between a low-energy $\sigma^{*}$ resonance state and a dipole-bound state. ${ }^{10,19}$ The presence of an apparent $A^{\prime}$ resonance at $1.45 \mathrm{eV}$ in our calculation thus provides additional support for their proposed mechanism.

Gianturco and Lucchese ${ }^{28}$ report two $A^{\prime}$ resonances in their calculation, one at $0.012 \mathrm{eV}$ with a width of $0.46 \mathrm{eV}$ and one at $10.4 \mathrm{eV}$ whose width is $0.87 \mathrm{eV}$. These may possibly correlate to the peaks we see at 1.45 and $8.5 \mathrm{eV}$, although the differences in both position and width are rather large. The calculation of Tonzani and Greene ${ }^{31}$ does not produce $A^{\prime}$ resonances.

\section{Elastic differential cross sections}

Figure 4 shows the elastic differential cross section (DCS) at selected energies from 0.1 to $20 \mathrm{eV}$, obtained by combining the scattering amplitudes from the polarized $A^{\prime \prime}$ calculation in basis $\mathrm{C}$ with those from the polarized $A^{\prime}$ calculation in basis B. Because we have not applied a dipole correction, the extreme forward DCS is certain to be underestimated, but we expect dipolar effects to grow less signifi-

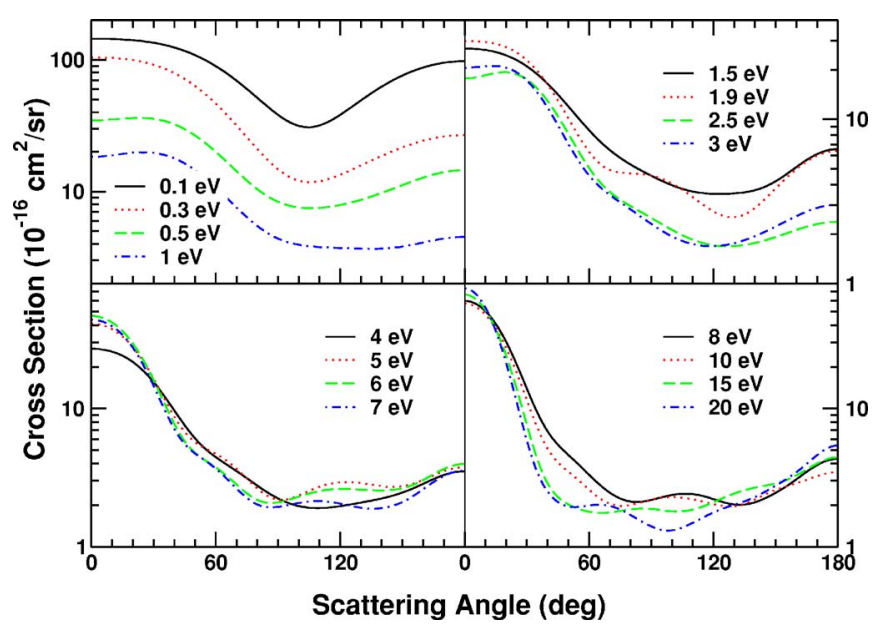

FIG. 4. (Color online) Differential cross sections for elastic scattering of electrons by uracil at selected energies.

cant as the scattering angle increases. Resonant effects on the DCS are visible at 1.9 and $5 \mathrm{eV}$, where, in each case, the angular pattern changes abruptly from that seen at neighboring energies. However, there is no evident signature of the lowest-energy $A^{\prime \prime}$ resonance in the DCS at $0.3 \mathrm{eV}$. At higher energies, the static-exchange DCS (not shown) generally agrees well with the DCS of Fig. 4, though the results obtained with polarization included tend to be somewhat more undulating at intermediate angles for energies below $\sim 15 \mathrm{eV}$ and are somewhat more backward-peaked at most energies.

\section{Excitation cross sections}

Integral cross sections for electron-impact excitation of the HOMO $\rightarrow$ LUMO transition are shown in Fig. 5. As discussed earlier, our thresholds, especially for the singlet state, are too high, so it would be reasonable to shift the cross sections downward to their correct thresholds, but we have not done so in plotting Fig. 5. Both the singlet and the triplet cross sections show a good deal of structure. While we expect the broad features of the cross sections to be meaning-

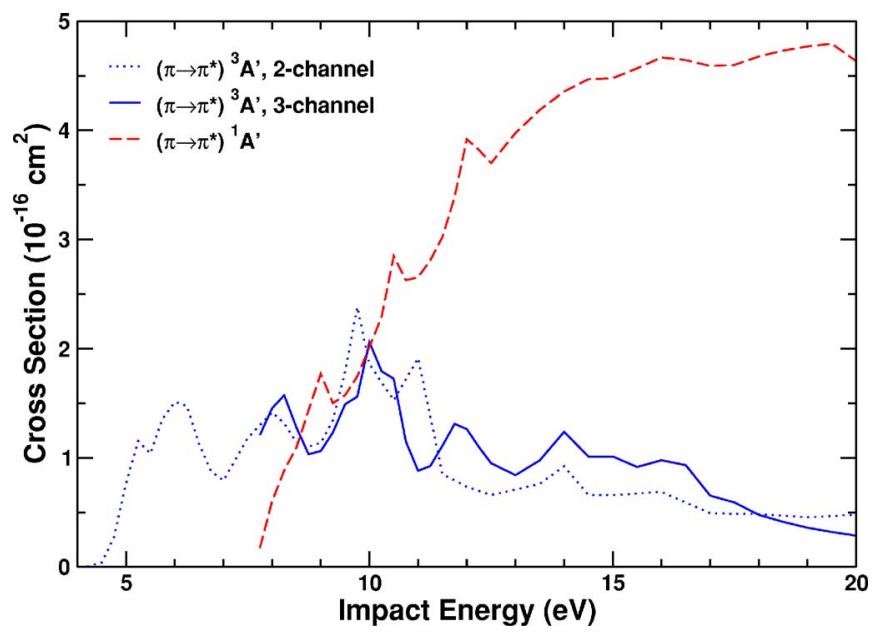

FIG. 5. (Color online) Cross sections for electron-impact excitation of uracil to the lowest triplet and singlet states of $A^{\prime}$ symmetry, associated with promotion of an electron from the highest occupied to the lowest unoccupied molecular orbital. 
ful, some or all of the small peaks may be spurious, arising from pseudoresonances associated with other channels that are treated as closed. Because the triplet channel has the lowest threshold of any excited state, and its cross section rises quickly from threshold to a significant value, it appears that triplet excitation will be a significant mechanism for energy deposition by slow electrons. The form of the singlet cross section, with a steady rise from threshold to a broad maximum, is typical for a dipole-allowed excitation, and the large value of the cross section reflects the significant oscillator strength carried by this $\pi \rightarrow \pi^{*}$ transition. Indeed, adding a Born-dipole correction ${ }^{45}$ for high-partial-wave scattering would increase the singlet cross section still further. We have not added such a correction to the cross section shown in Fig. 5 in part because it is less important near threshold than at energies well above threshold, and in part because comparison of our IVO-based oscillator strength (0.92 length, 0.42 velocity) with that obtained from a more sophisticated wave function (0.19, Ref. 38$)$ indicates that the dipolar contribution to our excitation cross section is already likely to be overestimated.

\section{SUMMARY AND CONCLUSIONS}

We have presented integral and differential elastic cross sections for elastic electron scattering by gas-phase uracil, as well as cross sections for excitation of the $1^{3} A^{\prime}$ and $2^{1} A^{\prime}$ electronic states. Our results place the first $A^{\prime \prime}$ resonance at $0.32 \mathrm{eV}$, in reasonable agreement with the energy, $0.22 \mathrm{eV}$, determined from electron-transmission experiments. ${ }^{10}$ Agreement is less close but still reasonable for the two higher $A^{\prime \prime}$ resonances. Additional features were found in both the $A^{\prime \prime}$ and the $A^{\prime}$ cross sections that persist from one calculation to another and therefore do not appear to be numerical artifacts. Although our current calculations do not supply sufficient information for us to be fully confident of assignments for these features, the peak at $1.45 \mathrm{eV}$ in $A^{\prime}$ symmetry appears to be a shape resonance and is in the appropriate energy range to be the $\sigma^{*}$ shape resonance invoked by Scheer and co-workers ${ }^{10,19}$ to explain the strong dissociativeattachment peak at $1.0 \mathrm{eV}$.

Although more limited in their treatment of polarization than is desirable, and thus not likely to give quantitative descriptions of the differential and integral cross sections, our calculations are likely to approximate resonance positions fairly well. Moreover, they may form a point of reference for future, higher-level calculations. We intend to revisit elastic scattering by uracil after making program improvements that will allow larger calculations, with more flexibility in both the one- and the many-particle basis sets, and more detailed analysis of their results.

\section{ACKNOWLEDGMENTS}

This work was supported by the U.S. Department of Energy, Office of Basic Energy Sciences, and employed computational resources of the Caltech-JPL Supercomputing Project.
${ }^{1}$ B. Boudaïffa, P. Cloutier, D. Hunting, M. A. Huels, and L. Sanche, Science 287, 1658 (2000).

${ }^{2}$ M. A. Huels, B. Boudaïffa, P. Cloutier, D. Hunting, and L. Sanche, J. Am. Chem. Soc. 125, 4467 (2003).

${ }^{3}$ M. A. Huels, I. Hahndorf, E. Illenberger, and L. Sanche, J. Chem. Phys. 108, 1309 (1998).

${ }^{4}$ K. Aflatooni, G. A. Gallup, and P. D. Burrow, J. Phys. Chem. A 102, 6205 (1998).

${ }^{5}$ R. Abouaf, J. Pommier, and H. Dunet, Int. J. Mass Spectrom. 226, 397 (2003).

${ }^{6}$ S. Denifl, S. Ptasinska, M. Cingel, S. Matejcik, P. Scheier, and T. D. Märk, Chem. Phys. Lett. 377, 74 (2003).

${ }^{7}$ G. Hanel, B. Gstir, S. Denifl, P. Scheier, M. Probst, B. Farizon, M. Farizon, E. Illenberger, and T. D. Märk, Phys. Rev. Lett. 90, 188104 (2003). ${ }^{8}$ R. Abouaf, J. Pommier, and H. Dunet, Chem. Phys. Lett. 381, 486 (2003).

${ }^{9}$ S. Denifl, S. Ptasińska, B. Gstir, P. Scheier, and T. D. Märk, Int. J. Mass Spectrom. 232, 99 (2004).

${ }^{10}$ A. M. Scheer, K. Aflatooni, G. A. Gallup, and P. D. Burrow, Phys. Rev. Lett. 92, 068102 (2004).

${ }^{11}$ S. Denifl, S. Ptasińska, G. Hanel, B. Gstir, M. Probst, P. Scheier, and T. D. Märk, J. Chem. Phys. 120, 6557 (2004).

${ }^{12}$ S. Ptasińska, S. Denifl, P. Scheier, and T. D. Märk, J. Chem. Phys. 120, 8505 (2004).

${ }^{13}$ S. Feil, K. Gluch, S. Matt-Leubner, P. Scheier, J. Limtrakul, M. Probst, H. Deutsch, K. Becker, A. Stamatovic, and T. D. Märk, J. Phys. B 37, 3013 (2004)

${ }^{14}$ R. Abouaf, J. Pommier, H. Dunet, P. Quan, P.-C. Nam, and M. T. Nguyen, J. Chem. Phys. 121, 11668 (2004).

${ }^{15}$ S. Denifl, P. Candori, S. Ptasińska, P. Limão-Vieira, V. Grill, T. D. Märk, and P. Scheier, Eur. Phys. J. D 35, 391 (2005).

${ }^{16} \mathrm{H}$. Abdoul-Carime, J. Langer, M. A. Huels, and E. Illenberger, Eur. Phys. J. D 35, 399 (2005).

${ }^{17}$ R. Abouaf and H. Dunet, Eur. Phys. J. D 35, 405 (2005).

${ }^{18}$ K. Aflatooni, A. M. Scheer, and P. D. Burrow, Chem. Phys. Lett. 408, 426 (2005).

${ }^{19}$ A. M. Scheer, C. Silvernail, J. A. Belot, K. Aflatooni, G. A. Gallup, and P. D. Burrow, Chem. Phys. Lett. 411, 46 (2005).

${ }^{20}$ S. Ptasińska, S. Denifl, B. Mróz, M. Probst, V. Grill, E. Illenberger, P. Scheier, and T. D. Märk, J. Chem. Phys. 123, 124302 (2005).

${ }^{21}$ S. Ptasinska, S. Denifl, P. Scheier, E. Illenberger, and T. D. Märk, Angew. Chem. Int. Ed. 44, 6941 (2005).

${ }^{22}$ P. D. Burrow, G. A. Gallup, A. M. Scheer, S. Denifl, S. Ptasinska, T. Märk, and P. Scheier, J. Chem. Phys. 124, 124310 (2006).

${ }^{23}$ R. Barrios, P. Skurski, and J. Simons, J. Chem. Phys. B 106, 7991 (2002).

${ }^{24}$ J. Berdys, I. Anusiewicz, P. Skurski, and J. Simons, J. Phys. Chem. A 108, 2999 (2004)

${ }^{25}$ J. Berdys, P. Skurski, and J. Simons, J. Phys. Chem. B 108, 5800 (2004).

${ }^{26}$ I. Anusiewicz, J. Berdys, M. Sobczyk, P. Skurski, and J. Simons, J. Phys. Chem. A 108, 11381 (2004).

${ }^{27}$ P. Możejko and L. Sanche, Radiat. Environ. Biophys. 42, 201 (2003).

${ }^{28}$ F. A. Gianturco and R. R. Lucchese, J. Chem. Phys. 120, 7446 (2004).

${ }^{29}$ P. D. Burrow, J. Chem. Phys. 122, 087105 (2005).

${ }^{30}$ A. Grandi, F. A. Gianturco, and N. Sanna, Phys. Rev. Lett. 93, 048103 (2004).

${ }^{31}$ S. Tonzani and C. H. Greene, J. Chem. Phys. 124, 054312 (2006).

${ }^{32}$ K. Takatsuka and V. McKoy, Phys. Rev. A 24, 2473 (1981); 30, 1734 (1984).

${ }^{33}$ C. Winstead and V. McKoy, Adv. At., Mol., Opt. Phys. 36, 183 (1996).

${ }^{34}$ C. Winstead and V. McKoy, Comput. Phys. Commun. 128, 386 (2000).

${ }^{35}$ M. W. Schmidt, K. K. Baldridge, J. A. Boatz, S. T. Elbert, M. S. Gordon, J. H. Jensen, S. Koseki, N. Matsunaga, K. A. Nguyen, S. J. Su, T. L. Windus, M. Dupuis, and J. A. Montgomery, J. Comput. Chem. 14, 1347 (1993).

${ }^{36}$ N. A. Oyler and L. Adamowicz, J. Phys. Chem. 97, 11122 (1993).

${ }^{37}$ J. D. Petke, G. M. Maggiora, and R. E. Christoffersen, J. Phys. Chem. 96, 6992 (1992).

${ }^{38}$ J. Lorentzon, M. P. Fülscher, and B. O. Roos, J. Am. Chem. Soc. 117, 9265 (1995).

${ }^{39}$ J. H. Hendricks, S. A. Lyapustina, H. L. de Clercq, J. T. Snodgrass, and K. H. Bowen, J. Chem. Phys. 104, 7788 (1996).

${ }^{40}$ C. Desfrançois, H. Abdoul-Carime, and J. P. Schermann, J. Chem. Phys. 104, $7792(1996)$ 
${ }^{41}$ C. Desfrançois, V. Periquet, Y. Bouteiller, and J. P. Schermann, J. Phys, Chem. A 102, 1274 (1998).

${ }^{42}$ S. D. Wetmore, R. J. Boyd, and L. A. Eriksson, Chem. Phys. Lett. 322, 129 (2000).

${ }^{43}$ S. S. Wesolowski, M. L. Leininger, P. N. Pentchev, and H. F. Schaefer III, J. Am. Chem. Soc. 123, 4023 (2001).

${ }^{44}$ X. Li, Z. Cai, and M. D. Sevilla, J. Phys. Chem. A 106, 1596 (2002).

${ }^{45}$ T. N. Rescigno and B. I. Schneider, Phys. Rev. A. 45, 2894 (1992), and references therein.

${ }^{46}$ T. N. Rescigno, D. A. Byrum, W. A. Isaacs, and C. W. McCurdy, Phys. Rev. A. 60, 2186 (1999).

${ }^{47}$ B. H. Lengsfield III, T. N. Rescigno, and C. W. McCurdy, Phys. Rev. A. 44, 4296 (1991).

${ }^{48}$ C. Winstead and V. McKoy, Phys. Rev. A. 57, 3589 (1998).

${ }^{49}$ C. Winstead, V. McKoy, and M. H. F. Bettega, Phys. Rev. A. 72, 042721
(2005).

${ }^{50}$ M. H. F. Bettega, C. Winstead, and V. McKoy, Phys. Rev. A. 74, 022711 (2006).

${ }^{51}$ C. W. Bauschlicher, J. Chem. Phys. 72, 880 (1980).

${ }^{52}$ M. J. Frisch, G. W. Trucks, H. B. Schlegel et al., Gaussian 98, Revision A.5 (Gaussian, Inc., Pittsburgh, PA, 1998).

${ }^{53}$ W. J. Hunt and W. A. Goddard, Chem. Phys. Lett. 3, 414 (1969).

${ }^{54}$ C. M. Marian, F. Schneider, M. Kleinschmidt, and J. Tatchen, Eur. Phys. J. D 20, 357 (2002).

${ }^{55}$ M. T. Nguyen, R. Zhang, P.-C. Nam, and A. Ceulemans, J. Phys. Chem. A 108, 6554 (2004).

${ }^{56}$ S. Huzinaga, J. Andzelm, M. Klobukowski, E. Radzio-Andzelm, Y. Sakai, and H. Tatewaki, Gaussian Basis Sets for Molecular Calculations (Elsevier, Amsterdam, 1984).

${ }^{57}$ B. Apagyi, P. Lévay, and K. Ladányi, Phys. Rev. A. 37, 4577 (1988). 